\title{
Use of Ontological Analysis as an Instrument of Knowledge Recognition in Complex Virtual Research Environments
}

\author{
Julia Rogushina $^{1}$, Anatoly Gladun ${ }^{2}$, Jeanne Schreurs ${ }^{3}$, Abdel-Badeeh Salem ${ }^{4}$ \\ ${ }^{1}$ Institute of Software Systems of National Academy of Sciences Ukraine, Ukrain \\ ${ }^{2}$ Center of Information Technologies and Systems of National Academy of Sciences, Ukraine \\ ${ }^{3}$ Department of Business Informatics, Hasselt University, Belgium \\ ${ }^{4}$ Department of Computer Science, Ain Shams University, Egypt
}

\begin{abstract}
Methods of ontological analysis for knowledge recognition in complex service-oriented virtual research environment are proposed. These methods provide acquisition of knowledge about domainspecific information objects (on example for $e$ learning domain - about qualification level and study domain of students and tutors). Reference domain ontologies are used as an instrument for student skills evaluation. Web services and ontologies provide reuse of knowledge in other applications. A prototype automatic tutor has been developed to support e-learning.
\end{abstract}

\section{Introduction}

Now information environment is an important factor of research and learning activities. The development of different virtual research environments with various features and services provide to user a lot of new functions and possibilities. But their efficiency depends of methods and algorithm developed for user tasks and specific problems.

Virtual Research Environments (VRE) are widely used for distributed knowledge management to be applied in different study domains. Knowledge management process for VRE can be organized in different ways that depend of domain and user tasks but all of them include the following steps: acquisition, creation, storage, validation, and utilisation of knowledge.

Knowledge management takes into account the structure and main elements of domain information objects.

An information object (IO) is a model of a real or virtual domain object (object, being, event, process, etc.) in the information space that defines the structure, attributes, constraints and, possibly, the behavior of this object [1]. For example, person, publication, Web-site, organization, city are objects, and their descriptions are information objects. By the Semantic Web conception such immaterial entities of the Web information space as ontologies, software agents, Web-services, information resources, metadata, and databases are IO too.
A lot of IO have some predetermined complex structure. Examples of such IO are organizations, educational institutions, people, Web-services, business processes. If we have some domain ontology then the appropriate classes of this ontology can be used as a resource of knowledge about domain IO structure, and then the values of these IO properties are acquired from the relevant IRs.

Information object is a main element of domain formalization that models information of material and virtual domain objects. If we use an ontological model of domain them information objects corresponds with classes and individuals of domain ontology. Relations between IOs represent connections between elements of domain model. For example, for e-learning domain main IOs are learner, tutor and qualification. In the most general learning is a process where tutor's qualification and skills are used for growth of learners qualification and skills. In this domain acquisition of knowledge about the learners' qualification level and skills is a complex problem.

This problem can be seen as a particular case of pattern recognition. The information object describes the qualification and the skills of the learners.

Computer technologies significantly changed the content and practice of education. E-learning is an alternative concept to the traditional tutoring system that offers new possibilities in learning [2]. In order to obtain better tutoring outcomes, a software tutoring system should emphasize engaging students in the learning process and be adaptive to each individual learner.

Ontological approach is a base for development of semantically enriched e-Learning systems: domain ontology can be used as a interoperable reused knowledge base. Thought the design of domain ontologies is an important part of the intelligent eLearning systems design.

An approach based on ontological knowledge representation is widely used for solving of object recognition problems on the semantic level. In our research we develop method that is based on reference domain ontology that is used as an instrument of evaluation of students' qualification 
and skills. The course ontology constructed by every student is matched with this reference ontology and all differences in concepts and relation are analyzed and rated. But another important problem is an environment that provides the use of this method for efficient e-learning process, the functions, services and possibilities of this environment.

\section{Virtual Research Environments}

Now a lot of researchers deal with generating new knowledge and cost-effective technologies offer a number of possibilities, which have not been exploited yet in Virtual Research Environments supported by e-infrastructures and methods and technologies of the Semantic Web, Computing, Networks, Artificial Intelligence etc. [3].

VRE platform provides re-use of existing relevant knowledge and solutions (e.g., tools and services from existing infrastructures and projects) at both European and national levels. Table 1 presents the challenges faced by the VRE implementation. The VRE platform manages data in such a way that their corresponding metadata semantics will be formally defined in a machine-understandable and interoperable manner.

Table 1. Challenge facing

\begin{tabular}{|l|l|}
\hline Challenge & Realization \\
integration & $\begin{array}{l}\text {-Adopting open data, open } \\
\text { across all } \\
\text { layers of the e- } \\
\text { infrastructure } \\
\text { main and open innovation as } \\
\text { implementing an advanced } \\
\text { dedicated software application to } \\
\text { facilitate e-infrastructure } \\
\text { networking resources integration. } \\
\text {-Encompassing physical e- } \\
\text { infrastructures and computing } \\
\text { models, including HPC, grid and } \\
\text { cloud computing models. } \\
\text { - Semantic annotation of data for } \\
\text { further semantic integration into } \\
\text { ontologies using standardized } \\
\text { ontological languages. } \\
\text { - Semantic Web services and } \\
\text { intelligent agents for integrating } \\
\text { software applications }\end{array}$ \\
\hline $\begin{array}{l}\text { Cross- } \\
\text { disciplinary } \\
\text { data interope- } \\
\text { rability }\end{array}$ & $\begin{array}{l}\text { these can be interoperated } \\
\text { amongst VRE services and users } \\
\text { overcoming possible } \\
\text { disciplinary-related } \\
\text { terminological discrepancies. } \\
\text { - Semantic Web services for } \\
\text { VRE-provided services and } \\
\text { resources decoupled with respect } \\
\text { to both the data provided by such }\end{array}$ \\
\hline
\end{tabular}

\begin{tabular}{|c|c|}
\hline & $\begin{array}{l}\text { services and the (user) services } \\
\text { requested }\end{array}$ \\
\hline $\begin{array}{l}\text { data citation, } \\
\text { sharing and } \\
\text { trust }\end{array}$ & $\begin{array}{l}\text { - semantically annotated } \\
\text { metadata for each data for those } \\
\text { ones further processing so that } \\
\text { they will include features like } \\
\text { authorship and source of } \\
\text { publication } \\
\text { - VRE platform will encompass } \\
\text { security mechanisms and } \\
\text { protocols against external attacks } \\
\text { - the use of ontologies and } \\
\text { Semantic Web services in } \\
\text { carrying out the main activities in } \\
\text { the VRE platform }\end{array}$ \\
\hline
\end{tabular}

They will support proof of concept, prototyping and deployment of advanced data services and environments, and access to top-of-the-range connectivity and computing.

\section{Ontological analysis and VRE}

Ontologies, which are commonly conceived as explicit formalizations of shared conceptual systems, are the most widely used approach to represent knowledge, due to their intrinsic properties of structure, reuse, sharing and formalization. All these properties enable them even for the automatic integration of knowledge once this has been represented [4].

Ontologies provide a common vocabulary of an area and define - with different levels of formality the meaning of the terms and the relations between them. Knowledge in ontologies is mainly formalized using classes, relations, functions, axioms and instances.

Concept "ontology" comes from philosophy. In state-of-the-art information technology (IT), an ontology is the working model of entities and interactions in some particular domain of knowledge or practices. In IT an ontology is the specification of conceptualizations, used to help programs and humans share knowledge. In this meaning, an ontology consists of specified concepts that are defined to create an agreed-upon vocabulary for information exchange.

Knowledge in ontologies is formalized on base of five main kinds of components: classes, relations, functions, axioms and instances. T. Gruber defines the ontology as a declarative knowledge description that contains classes (concepts) and relationships between them. [5].

Ontological analysis aims the development of the vocabulary that is used to discuss the characteristic objects and processes that compose the domain, developing definitions of the basic concepts in that vocabulary, and characterizing the logical connections among those terms. 
The product of this analysis, an ontology, contains domain vocabulary complete with a set of precise definitions (axioms) that constrain the meanings of the terms sufficiently to enable consistent interpretation of the data that use that vocabulary and relations between concepts from vocabulary.

The ontological analysis - the process of development of ontologies involves several stages associated with specification of different components of ontology:

- $\quad$ the area and scope of the ontology;

- options to reuse existing ontologies;

- important for ontology terms ;

- classes and class hierarchies;

- classes - slots properties;

- facet properties;

- instances.

The ontology is knowledge base of a special kind with the semantic information about some domain. It is a set of definitions in some formal language of declarative knowledge fragment focused on joint repeated use by the various users in the applications.

The most known languages of ontology representation are KIF (Knowledge Interchange Format), DAML + OIL (DARPA Agent Markup Language) and OWL (Ontology Web Language). Today the most popular is OWL and its last modification OWL 2.0.

Ontology built on OWL is a sequence of axioms and facts with the addition of references to other ontologies associated with it. OWL is based on a logical model that allows to give a definition of the concepts as they are described and to use the mechanism of reasoning.

The knowledge in ontologies is mainly formalized by use of five kinds of components: classes, relations, functions, axioms and instances.

Formal model of ontology $\mathrm{O}$ is ordered triple of finite sets

$\mathrm{O}=\langle\mathrm{T}, \mathrm{R}, \mathrm{F}\rangle$

where

- $\mathrm{T}$ - final set of the terms of domain that is described by ontology $\mathrm{O}$;

- $\quad \mathrm{R}$ - final set of the relations between the terms of given domain from $\mathrm{T}$;

- F - final set of functions of interpretation given on the terms $\mathrm{T}$ and relations $\mathrm{R}$ of ontology $\mathrm{O}$.

Relations that represent a type of interaction between concepts of the domain are formally determined as any subset of products (crossings) of sets n, such as:

$\mathrm{R}: \mathrm{C} 1 \times \mathrm{C} 2$ x... x Cn.
Examples of the binary relations: "A is a subclass of B" and "A is connected with B". In [6] the most common relations in real domains are discriminated. The most common binary relations that are used in real domain ontologies can be discriminated on following categories:

- connection of equivalence;

- taxonomic connection;

- $\quad$ structural connection;

- connection of dependence;

- topological connection;

- connection of the reason and consequence;

- functional connection;

- chronological connection;

- connection of similarity;

- conditional connection;

- $\quad$ target connection.

Till now no generally accepted universal definition of domain ontology has been suggested. In [7] different definitions are analyzed. On the meaningful level a domain ontology will be understood as a set of agreements (domain term definitions, their commentary, statements restricting a possible meaning of these terms, and also a commentary of these statements). Domain ontology is:

- the part of domain knowledge that is not to be changed;

- the part of domain knowledge that restricts the meanings of domain terms;

- a set of agreements about the domain;

- an external approximation represented explicitly of a conceptualization given implicitly as a subset of the set of all the situations that can be represented.

All these meanings of the notion of domain ontology supplement each other.

\section{Research and Innovation Activities of the VRE}

The following main types of research- and innovation- activities, covering a variety of research topics about the trans-disciplinary nature of the VRE, have been linked to the problem and the resulting solution.

High Performance Computation (HPC) is set forward. The current e-infrastructure services related to HPC, Grid and Cloud, which have been funded by national or European funding agencies, are focused on computational intensive services, rather than on data processing [8].

As underlined in the PRACE report ("The scientific case for high-performance computing in 
Europe 2012-2020"), handling large data volumes generated by research is a major challenge and opportunity for future HPC systems and integrated environments for computing and data management. SMART-VRE intends to provide a showcase of an integrated environment that can serve a specific community, the one engaged in ageing research.

Since specialized data services are becoming complex and expensive to maintain by datacenter management, a recent trend is their deployment in Private or Public Clouds. The migration and deployment is nowadays not straightforward and requires specific knowledge and manual intervention [9].

Networking, or co-sharing computing services, is fostering forms of shared information thanks to the engagement of agents and resources improving participatory approaches and direct involvement.

Open Science refers to dynamic systems of knowledge production, characterized by a more or less high degree of accessibility of information and by knowledge of researchers and scientists. These systems act as dynamos, generators and stimulators of knowledge for future research. Open Science implies the creation of effective networks based on shared collaborative resources using technical tools that are able to distribute the information. The collaborative technologies are facilitating also the distribution resources including protected data (proprietary data and materials, trade secrets, legal protections, intellectual property etc.).

The Open Innovation [10] concept is one of the central aspects of the processes of diffusion of innovation and technology transfer.

In general, Open Innovation can be defined as the result of the use of purposive inflows and outflows of knowledge to accelerate internal innovation, and to expand the markets for external use of innovation. In literature, several international case studies are cited from which it is possible to understand the concrete operation of these processes and to identify the most important factors involved.

Both concepts of open innovation and of open science will guide the high-level strategy to carry out the networking activities in SMART-VRE (see Figure 1).

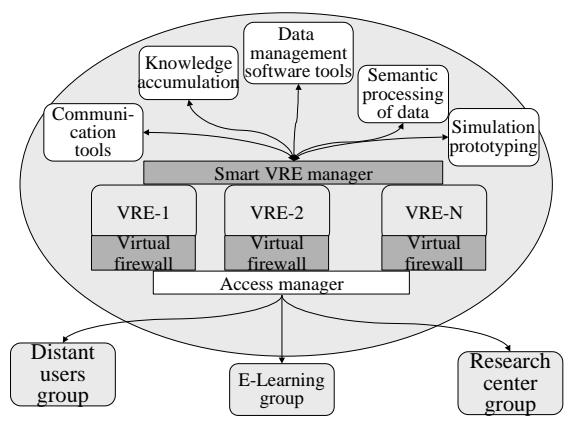

Figure 1.The VRE Framework
To ensure the exploitation of data, data must be available and accessible in a network environment. However, the nature of data (research, administrative, academic) is variable and dependent of the scientific discipline, the application scope and the life cycle. A critical point in data management is the metadata representation of datasets catalogs [11]. The correct management of research data is a fundamental part of the research process. This management involves making decisions and actions before the creation of the data, during its creation and use and throughout its life cycle.

\section{Semantic Web technologies and e- Learning}

Ontologies and reasoning techniques are leading to the achievement of a more intelligent Web or to the automation of science. The purpose of the Semantic Web (SW) [12] is to add semantics to the data on the Web (for example, establish the meaning of the data using metadata), so that machines can process these data like humans can do. In order to achieve this aim, ontologies are expected to be used to provide structured vocabularies that describe the relationships between different concepts, allowing computers (and humans) to interpret their meaning in a flexible way and unambiguously. Although there are several ontological languages, OWL [13] is the de facto Semantic Web standard ontology language. Semantic Web proposes more intelligent services by facilitating machine understanding of content that can be used for different VRS purposes. Ontologies are an important building block of knowledge in the Semantic Web that are supported by it's technologies and standards. Ontological representation of knowledge provides a shared and common understanding of a domain that can be communicated across people and applications.

Semantic Web technologies in the context of elearning provide deeper understanding of the relevant issues. The following pairs where the first part relates to the Semantic Web key issue and the second one to the e-learning key issue demonstrate it:

- Ontological evolution and Adaptive hypermedia: traditional adaptive hypermedia in e-learning have been combined with ontological engineering, and ontology construction, ontology integration, conceptual modeling and semantic conceptualization reveal a new research agenda, in which the specifications of conceptualizations (ontologies) promote the performance of learning systems.

- Expression of meaning and Content authoring combines the traditional content authoring process with the critical objective of expression 
of meaning: semantic mark-up, semantic retrieval, personalized structured annotation and content conversion are the parts of big research stream where the main concern is the development of semantic e-learning content.

- Information flow and collaborative e-Learning context. The instrumentation of knowledge flows has been included in the priorities of the $\mathrm{W} 3 \mathrm{C}$ Semantic Web activity. Within this area, semantic services, (semi-) automated reasoning and argumentation are critical themes on the semantic e-learning agenda.

- Policy-aware infrastructure and Standards of Interoperability: e-learning industry demonstrate many achievements within the area of interoperability and standards, and from this perspective this industry recognizes the need to secure a policy-aware infrastructure. The Semantic Web provides an information space for the free flow of scientific and cultural information if its infrastructure supports a full range of fine-grained policy controls over the information it contains. All these researches influence VRE development. The issues of different types of control over content, the compliance to semantic and metadata models, as well as the versioning and provenance of content, require extensive research.

On the other hand, the multi-agent systems and intelligent agents area has received increasing attention by researchers since the end of last century and is currently very SW-relevant. An „Agent” could be defined as a computer system situated in some environment and capable to action autonomously in this environment in order to meet its design objectives. Agents having reactivity (i.e. the ability to perceive its environment and respond to changes to it in a timely fashion), pro-activeness (i.e. the ability to exhibit goal-directed behavior by taking the initiative), and social ability (i.e. the ability to interact with other agents) have been called as the weak notion of agency. Intelligent agents can exhibit some other properties such as temporal continuity (i.e. an agent functions continuously and unceasingly), reasoning (i.e., decision-making mechanism, by which an agent decides to act on the basis of the information it receives, and in accordance with its own objectives to achieve its goals), rationality (i.e. an agent's mental property that attract it to maximize its achievement and to try to achieve its goals successfully), veracity (i.e. mental property that prevents an agent from knowingly communicating false information), mobility (i.e. the ability for a software agent to migrate from one machine to another), etc.

In particular, one main characteristic of an agent is the learning ability, that is, the capacity to adapt or modify its behavior based on learning experiences.
Agents can be useful as standalone entities that are delegated particular tasks on behalf of a user. However, in the majority of cases, agents exist in environments that contain other agents, constituting Multi-agent Systems (MAS). MAS can be seen as a group of agents that can potentially interact with each other. MASs present several advantages over isolated agents, such as reliability and robustness, modularity and scalability, adaptively, concurrency and parallelism, and dynamism.

Learning should also be a fundamental capability as a way to keep track of the changes in VRE users preferences [14]. Argumentation has been gaining increasing importance, mainly as a vehicle for facilitating rationally justifiable decision making when handling incomplete and potentially inconsistent information. The joint application of Semantic Web and Web Services in order to create intelligent Web Services is referred to as Semantic Web Services (SWS). SWS consists of describing Web Services with semantic content so that service discovery, composition and invocation can be done automatically.

The W3C has examined various approaches with the purpose of reaching a standard for the Semantic Web Services technology, including OWL-S, WSMO, SWSF, WSDL-S, and the proposed as W3C recommendation, SAWSDL. The first three approaches propose an ontology that semantically describes all relevant aspects of Web Services. On the other hand, WSDL-S and SAWSDL identify some WSDL and XML Schema extension attributes that support the semantic description of WSDL components.

\section{Use of reference domain ontology in E- learning as an object of evaluation}

E-tutor, supporting learners of an e-learning course, is an alternative concept to the traditional tutoring system. The course tutor in a software tutoring system controls learners relatively weaker than in the traditional one where it is the tutor who is in charge of the support of learning content and fulfilling the assignments. Therefore, in order to obtain better tutoring outcomes, a software tutoring system should emphasize engaging students in the learning process and be adaptive to each individual learner. E-learning offers new possibilities for the learner. The learner can get immediate feedback on his solved problems, can have individualized learning paths, etc.

E-learning services business is growing. The number of organizations working on E-learning and delivering e-learning tools with varying functionality is growing. The number of e-learning courses on the Internet is increasing rapidly. A structured information representing is required and ontologies 
(machine process representation containing the semantic information of a domain) can be very useful. The ontology systems serve as a flexible and extendable platform for e-learning management. For example, the expediency of computer ontologies can be used as a transparency tool of European and national qualification frameworks is reasoned. Qualifications are described by triads of professional qualities - knowledge, skills and competencies. A model oriented training helps to compare qualifications and simplifies the procedure for their acceptance.

One of the main problems arising during creation of testing systems is an interoperability of created tests - opportunity to reuse these tests in different testing systems. To organize test exchange between various systems it is necessary to create some universal format of tests preservation and their processing instructions. And an important condition for this format should be its independence from the platform. Standardization of educational technologies and, in particular formats of test data preservation is working out all over the world. Now Ministry of Education and Science of Ukraine realize the Program of On-line Education Development.

According to these activities the development of projects of standards for systems, methods and technologies standards of on-line education in educational institutions taking into account international standards was provided. But different test formats such as Instructional Management Systems (IMS) Question and Test Interoperability (QTI) of Global Learning Consortium are not adequate for the representation of all domain relations.

The more serious problems are caused by the semantic testing. Many authors use the ontology's semantic data to improve the analyses of information in unstructured documents. The domain ontology plays a central role in resource structuring of the learning content. One of the key challenges of the course construction process is to identify the abstract domain of information within which this course will exist. The tutor has to describe the main terms and concepts from which a course is to be constructed.

The main idea of our approach is that the domain ontology is not only the instrument of learning but an object of evaluation of students. We propose for students to build the domain ontology of the study domain and then compare it with the reference one. Results of this comparison show the parts of the domain knowledge which were wrong understood by the student and will help the tutor to improve the elearning course.

Realized experiments demonstrate that this approach is much more efficient then usual tests where some mistakes can be involved by ambiguous formulation of questions and misprints, but correct answers can be obtained intuitively or by accident and don't reflect the real student understanding of the concept about the domain.

We consider that a professional activity is a characteristic of a domain. This activity consists in solving different tasks. Task solving needs special knowledge, the same for all the tasks, that can be represented verbally. Therefore we can speak about special vocabulary of every domain that is used for specification of tasks and their solutions in this domain. A domain is considered as a set of the tasks, which are solved by specialists of this domain. When solving a task, a person uses a finite set of objects and relations among them.

In the process of ontology building, students use relations from the fixed set that contains the most widely used relations: $\mathrm{R}=\{$ "is a subclass of", "is a part of", "is a synonym", "has attributes", "has elements" \}. It simplifies the ontology building and analyses processes. The students (as well as the tutor) have to execute four main steps to design the ontology of domain:

1. Define the main classes and terms of the domain and describe their meaning: the set of class names $\mathrm{T}$; the set of relation names $\mathrm{R}$;

For every class name define the set of attribute names At; for every attribute name $a \in A_{t}, t \in T$ define its type - INT, STRING, NUMBER ets. or other class of ontology;

2. Construct the taxonomy of domain terms:

$<\mathrm{t}_{1}, \mathrm{t}_{2}>, \mathrm{t}_{1} \in \mathrm{T}, \mathrm{t}_{2} \in \mathrm{T}, \mathrm{r}\left(\mathrm{t}_{1}, \mathrm{t}_{2}\right)->$

$\mathrm{t}_{1}$ "IS_A_Subclass_Of" $\mathrm{t}_{2}, \mathrm{r} \in \mathrm{R}$

3. Define synonymy and other relations:

$<\mathrm{t}_{1}, \mathrm{t}_{2}>, \mathrm{t}_{1} \in \mathrm{T}, \mathrm{t}_{2} \in \mathrm{T}, \mathrm{r}\left(\mathrm{t}_{1}, \mathrm{t}_{2}\right)->$;

$\mathrm{t}_{1}$ "IS_Synonyme_Of" $\mathrm{t}_{2}, \mathrm{r} \in \mathrm{R}$

$<\mathrm{t}_{1}, \mathrm{t}_{2}>, \mathrm{t}_{1} \in \mathrm{T}, \mathrm{t}_{2} \in \mathrm{T}, \mathrm{r}\left(\mathrm{t}_{1}, \mathrm{t}_{2}\right)->$;

$\mathrm{t}_{1}$ "Re lated_With" $\mathrm{t}_{2}, \mathrm{r} \in \mathrm{R}$

4. Describe the instances of constructed classes $\forall \mathrm{a} \in \mathrm{t}, \mathrm{t} \in \mathrm{T}$.

\section{Comparison of ontologies}

The tutor compares domain ontologies built by students with reference ontology constructed by tutor. The main feature of them that simplifies their matching is the use of the same fixed sets of terms and relations (these set are imported from the reference ontology).

In process of matching of two similar but different knowledge structures some types of differences can be appear:

- Hierarchical (differences in relations "classsubclass" between concepts;

- Structural (other differences in relations between concepts); 
- Classification (differences in relations "classindividual").

We use the original algorithm for automatically comparing of ontologies that provides correspondence of hierarchical levels in term taxonomy (if class $\mathrm{A}$ is a subclass of $\mathrm{B}$ in reference taxonomy and $\mathrm{B}$ is a subclass of $\mathrm{A}$ in students taxonomy there is a mistake) and controls affiliation of instances with classes (if instance a belongs to class $\mathrm{A}$ in reference taxonomy and student describe instance a that belongs to class B is a mistake).

This algorithm is based on some specific conditions and that's why can't be used for matching of arbitrary ontologies:

- Student has to use ontological terms for classes and subclasses only from the fixed set corresponding to terms of reference ontology, other terms are considered as mistakes;

- Student has to use relations between classes only from the fixed set corresponding to relations of reference ontology, other relations are considered as mistakes too;

- If student nevertheless use some term that doesn't exist in reference ontology this term has to correspond to some term of reference ontology (student can use the incorrect name by mistake)

\section{Algorithm of ontology comparison}

We compare the student ontology $\mathrm{O}_{\mathrm{s}}$ with reference ontology $\mathrm{O}_{\mathrm{e}}$ made by tutor

1. Define the sets of ontology terms Ts and Te .

2. Classify terms from $\mathrm{Ts}$ on three disjoint categories $T n, T u$ and Tw. $T_{s}=T_{n} \cup T_{u} \cup T_{w}$ where

- Correctly defined terms $T_{n} \subseteq T_{e}$,

- Not accurately defined terms $\mathrm{T}_{\mathrm{u}} \not \subset \mathrm{T}_{\mathrm{e}}$ but $\forall \mathrm{t}_{\mathrm{i}} \in \mathrm{T}_{\mathrm{n}} \exists \mathrm{t}_{\mathrm{j}_{1}} \in \mathrm{T}_{\mathrm{e}}, \ldots, \mathrm{t}_{\mathrm{j}_{\mathrm{m}}} \in \mathrm{T}_{\mathrm{e}}, \mathrm{t}_{\mathrm{j}_{\mathrm{k}}} \in \mathrm{T}_{\mathrm{e}}, \mathrm{m}=\overline{1, \mathrm{k}}$ , and

- Incorrectly defined terms $\mathrm{T}_{\mathrm{u}} \not \subset \mathrm{T}_{\mathrm{e}}$ and $\forall \mathrm{t}_{\mathrm{i}} \notin \mathrm{T}_{\mathrm{n}} \neg \exists \mathrm{t}_{\mathrm{j}} \in \mathrm{T}_{\mathrm{e}}$.

3. Define the sets of ontology relations $\mathrm{Rs}$ and $\mathrm{Re}$.

4. Classify relations from $\mathrm{Rs}$ on three disjoint categories: $\mathrm{Rn}, \mathrm{Ru}$ and $\mathrm{Rw}$. $\mathrm{R}_{\mathrm{s}}=\mathrm{R}_{\mathrm{n}} \cup \mathrm{R}_{\mathrm{u}} \cup \mathrm{R}_{\mathrm{w}}$ where

- Correctly defined terms $\mathrm{R}_{\mathrm{n}} \subseteq \mathrm{R}_{\mathrm{e}}$,

- Not accurately defined terms $\mathrm{R}_{\mathrm{u}} \not \subset \mathrm{R}_{\mathrm{e}}$ but $\forall \mathrm{r}_{\mathrm{i}} \in \mathrm{R}_{\mathrm{n}} \exists \mathrm{r}_{\mathrm{j}_{1}} \in \mathrm{R}_{\mathrm{e}}, \ldots, \mathrm{r}_{\mathrm{j}_{\mathrm{m}}} \in \mathrm{R}_{\mathrm{e}}, \mathrm{r}_{\mathrm{j}_{\mathrm{k}}} \in \mathrm{R}_{\mathrm{e}}, \mathrm{m}=\overline{1, \mathrm{k}}$ , and

- Incorrectly defined terms $\mathrm{R}_{\mathrm{u}} \not \subset \mathrm{R}_{\mathrm{e}}$ and $\forall \mathrm{r}_{\mathrm{i}} \notin \mathrm{R}_{\mathrm{n}} \neg \exists \mathrm{r}_{\mathrm{j}} \in \mathrm{R}_{\mathrm{e}}$.
5. Analyze the use of ontology terms and relations. We don't consider the use of terms from Tw and relations from Rw. It is very important to take into account the type of relations - hierarchical or improper: Mistake of use "is a part" relation instead of "is a subclass" is much less principle then use "is a synonym" relation instead of "is a subclass" one.

\section{Classification of mistake types in student ontologies}

Different ontological relations have their own semantics and weight that can be used in evaluation of student ontologies and their mistakes. Elements of the mereological analysis applied to modern means of ontological analysis are used for it [15]. Examples of the binary relations: "A is a subclass of $\mathrm{B}$ " and "A is connected with B". However not all these ontological relations are equal in value. There is possible to allocate some fundamental relations in this set, such as taxonomy and mereology. Their weight is bigger because these relations define more important characteristics of domain. So we can evaluate different mistakes of student ontology that deal with relations of different types with various weights.

Domain relation is named fundamental if on the basis of this relation the formal system allowing to express the basic mathematical concepts of domain can be constructed. There are four fundamental relations, on the basis of each of which the mathematics can be constructed:

- the relation of belonging (set theory ZF and NF),

- the relation between function, its argument and result (fon Neumann set theory),

- the naming relation (ontology of Lesnevsky),

- the relation "a part of" (mereology).

Taxonomy in general is usually defined as a system of classification. The word "taxonomy" comes from two Greek words: "taxis" (order) and "nomos" (agreement). A typical example of taxonomy is the hierarchical tree. The taxonomy is an instrument for sharing into the ordered groups or categories. So ontology can be represented as "polytaxonomic" structure. Term is borrowed from philosophy. From the ontological point of view, taxonomy is the ontological organization based on the partial ordered relation called " $\mathrm{x}$ is $\mathrm{A}$ » by means of which the objects are grouped together or more high level is referred to classes. The classes in ontology are usually organized in the taxonomy by the relation "class-subclass" but any object can belong to more than one subclass. The term "taxonomy" has a widespread but the term "mereology" in researches connected with IT is applied more occasionally and that is why requires an additional description of this relation. 
Mereology (from two Greek words: "meros" (part) and "logy" (study, discussion, science) is a formal theory about parts and concepts connected to them. Mereology has been explored in various ways as applications of predicate logic to formal ontology, in each of which mereology is an important part. Each of these fields provides their own axiomatic definition of mereology. This term was proposed by the Polish philosopher Lesnevsky who had analyzed philosophical, logic and mathematical components of the mathematics bases. The calculus of names of Lesnevsky is constructed as alternative to logic system of Rassel for purposes of natural generalization of traditional logic. The mereologic relation "part - whole" is extremely important because it forms a concept basis of system that is central in modern scientific knowledge [16].

Among the mereological relations it is possible to allocate seven classes, and in general, the transitivity is not accepted among instances of different classes:

1. Component - object: page - book;

2. Member - collection: tree - wood;

3. Part-weight: piece - bread;

4. Material - object: aluminum - airplane;

5. Property - activity: to see - to read;

6. Stage - process: boiling - preparation of tea;

7. Place - area: Ukraine- Europe.

We distinguish the mistakes of different gravity. If student uses improper relation but from group of hierarchical relations (for example, $\mathrm{A}$ is a part of $\mathrm{B}$ instead of $\mathrm{A}$ is a subclass of $\mathrm{B}$ - see Figure 4) it is not so important as if she or he uses hierarchical relation instead of synonymic relation (for example, $A$ is a part of B instead of A is a synonym of B). More serious mistake is improper direction of hierarchical relations (for example, $\mathrm{A}$ is a part of $\mathrm{B}$ instead of B is a part of A).

\section{Sources of reference ontologies}

Reference ontology of course can be constructed by tutor especially for qualification evaluation or imported from other intelligent applications deal with this course or with course domain. The most adequate variant is an integration of both these methods with use of standardized discipline and specialty curriculums and other methodical materials. For example, computer ontologies for description of qualification 3121 "Information technology specialist" can be built. This field of knowledge is determined by the world community as "Computing" and represents a generalized knowledge of the branch which includes computer engineering, computer sciences, software engineering, information systems and information technology. In the Ukrainian educational system the branch of knowledge "Computing" is represented by branches 0403 "System sciences and cybernetics" (training direction 040301 Applied Mathematics,
040302 "Informatics" and 040303 System Analysis), 0501 "Informatics and Computer Engineering" (training direction 050101 "Computer Sciences", 050102 "Computer Engineering" and 050103 "Software Engineering") and 0502 "Automation and Management" (training direction 050201 "System Engineering" and 050202 "Automation and computer-integrated technologies").

In this case the unified representation of information on standards of higher education in a particular field of knowledge in the form of the ontological model is considered to be absolutely logical.

By describing of all the classes and properties of the objects related to this subject domain in accordance with the branch standard of higher education, we obtain the ontology which semantically represents this qualification and its courses. At the next step this domain ontology can be complemented by the description of the levels of the European and National Qualifications Frameworks and their components (knowledge, skills, and competencies).

An important instrument for integration of different ontologies deal with e-learning is a general competence ontology that defines high-level terms of learning domain. We specially design this ontology for definition of the most important terms such as "discipline", "competence", "tutor", "student", their properties and relations (see Figure 2).

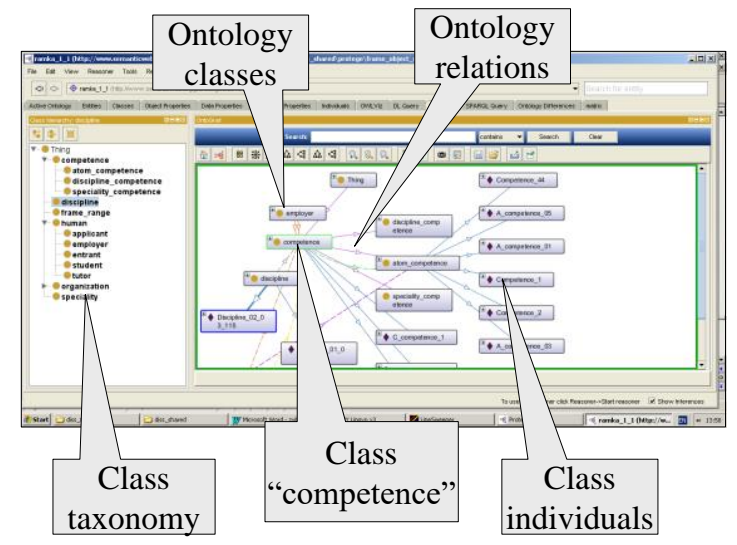

Figure 2. The competence ontology

\section{Knowledge acquisition from natural language documents}

In some situations students are not able to build the domain ontology (for example, if they are specialized in sphere not relevant to information technologies they don't know the basis of ontological analysis). In this case we need to build an ontology that contains student's knowledge automatically. As a source of this knowledge we can use all natural language texts generated by this student - written tests, reports etc. It is possible with use of sets of 
terms and relations from reference ontology proposed by tutor. The structure of the domain ontology is based on reference domain ontology. Domain is represented by the "lightweight" ontology containing no axioms. This greatly simplifies its use and provides faster performance algorithms analysis. On the base of reference domain ontology the lexical ontology that connects domain ontology terms and relations with fragments of natural language text is built. Every ontology term is connected with the set of corresponding natural text fragments. Lexical ontology is a set of pairs:

- reference ontology terms and relevant fragments

$$
\text { of } \operatorname{NLP}\left\langle\mathrm{t}_{\mathrm{i}} \in \mathrm{T}_{\text {ref }}, \mathrm{s}_{\mathrm{i}_{\mathrm{j}}}\right\rangle \text {; }
$$

- reference ontology relations and relevant fragments of NLP $\left\langle\mathrm{r}_{\mathrm{k}} \in \mathrm{R}_{\mathrm{ref}}, \mathrm{s}_{\mathrm{k}_{\mathrm{j}}}\right\rangle$;

- reference ontology individuals and relevant fragments of $\operatorname{NLP}\left\langle\mathrm{o}_{\mathrm{i}_{\mathrm{m}}} \in \mathrm{t}_{\mathrm{i}} \in \mathrm{T}_{\text {ref }}, \mathrm{s}_{\mathrm{i}_{\mathrm{m}}}\right\rangle$.

This ontology can be used for semantic markup of natural language texts and is exploited for processing of student's texts. On base of this markup we can automatically analyze the domain relations that student believes between the domain concepts and instances [17].

Stage 1. The set of terms is exported from the reference ontology.

Stage 2. Lexical ontology is built (automatically or by tutor).

Stage 3. Students` natural language text are marked up by reference ontology terms and relations (with the help of lexical ontology).

Stage 4. Students' beliefs about course domain are acquired from this markup by the following rules:

- Rule 1. If two fragments from one sentence are marked up by concepts of reference ontology A and $\mathrm{B}$ and some fragment is marked by relations $\mathrm{X}$ from the reference ontology then we can check the semantics of this sentence.

- Rule 2. If reference ontology contains relation X between concepts $\mathrm{A}$ and $\mathrm{B}, \mathrm{X}(\mathrm{A}, \mathrm{B}) \in \mathrm{O}_{\text {reference }}$, then we can assume that student correctly understands this regularity.

- Rule 3. If reference ontology contains other relation $\mathrm{Y}$ (or have no relations) between concepts $\mathrm{A}$ and $\mathrm{B}$ then we analyze the type of $\mathrm{X}$ and $\mathrm{Y}$ relations. If $\mathrm{X}$ and $\mathrm{Y}$ are incompatible, $\mathrm{Y}(\mathrm{A}, \mathrm{B}) \in \mathrm{O}_{\text {reference }} \Rightarrow \mathrm{X}(\mathrm{A}, \mathrm{B}) \notin \mathrm{O}_{\text {reference }}$ then we can assume that student incorrectly understands this regularity.

We also analyze relations of subclasses and superclasses of A and B if both have relation $\mathrm{X}$,

$$
\exists X\left(\begin{array}{l}
A^{`} \in \operatorname{subclass}(A) \cup \sup \operatorname{erclass}(A), \\
B^{`} \in \operatorname{subclass}(B) \cup \sup \operatorname{erclass}(B)
\end{array}\right) \in O_{\text {reference. }}
$$

Stage 5. We can assume that student understands this regularity but with some mistakes.

This algorithm provides analysis of ontological classes and individuals as well. In natural language the equivalents of individuals are named entities (names, titles etc.). If reference ontology includes individuals we can analyze the relations of concepts and their individuals.

\section{The implementation of the prototype}

Ontological representation of student domain skills can be automatically processed by intelligent software agents. Software agents of multi-agent system (MAS) for e-learning work efficiently in dynamic heterogeneous distributed environment by cooperation, coordination and negotiation techniques. A lot of researchers use MAS for elearning and e-coaching tasks [18].

$\mathrm{M}(\mathrm{e}) \mathrm{L}$ prototype is a multi-agent ontology-based e-learning system that produces automatic semantic control of student learnt course beliefs [19]. The focus of ontology analysis is on knowledge structuring (of main domain terms and their relations). General architecture of this system is proposed in Figure 3.

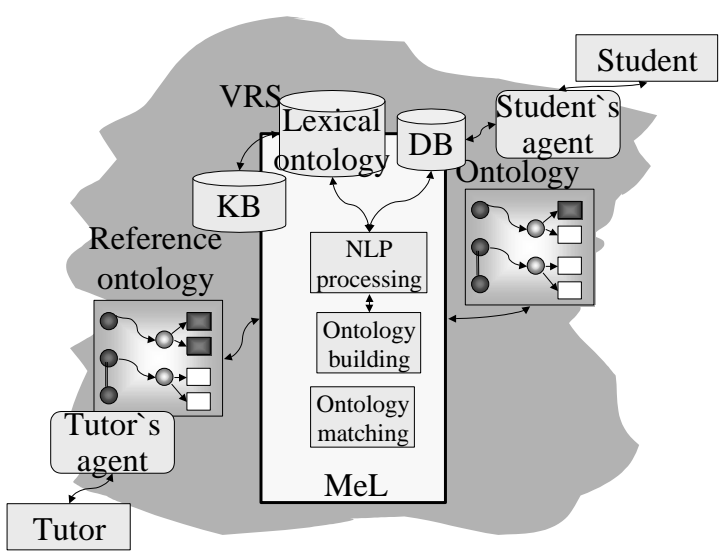

Figure 3. Ontology building process as a result of learning

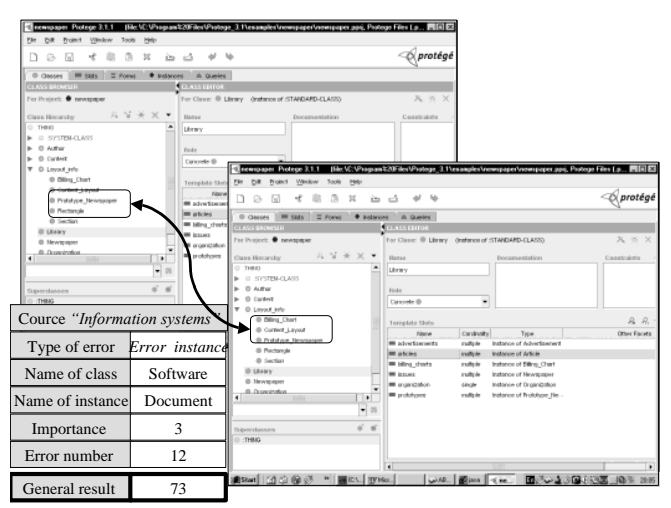

Figure 4. Domain ontology matching in $\mathrm{M}(\mathrm{e}) \mathrm{L}$ 
All $\mathrm{M}(\mathrm{e}) \mathrm{L}$ main function deal with knowledge processing are realized with the help of VRE services. We use ontologies to describe learning materials and to represent student's belief about the course domain (see Figure 4). Domain ontology is matched with reference one in $\mathrm{M}(\mathrm{e}) \mathrm{L}$. The errors are process with their weight.

\section{Conclusion and Future work}

Ontological representation of student domain skills can be automatically processed by intelligent software agents as we show by $\mathrm{M}(\mathrm{e}) \mathrm{L}$ prototype. The main features of our approach to knowledge control are the following: all results are analyzed automatically without human tutor, the results are analyzed objectively, students can work with knowledge base, a structuring of domain knowledge simplifies the learning process and tutors can exchange their knowledge based on reference ontologies.

Innovation of this approach deals with the role of domain ontology that is used as an instrument of analyzing of student beliefs. We suppose that modern information technologies can provide appropriate tools and methods for ontological analysis oriented not only on specialists in knowledge management sphere but on students of various specialties. Provided experiments confirm this assumption. In future we plan to develop the specialized means for ontology retrieval, development and matching that would be oriented on use in VREs and processing of semantically marked resources and information objects of the Semantic Web.

\section{Acknowledgment}

This work was supported in part by the project "Design of intelligent system of informational and cognitive support of National qualification frame functioning" of Melitopol State Pedagogic University.

\section{References}

[1] A. Gladun, J.Rogushina, R. Valencia-García, and R. Martínez-Béjar, "Semantics-driven modelling of user preferences for information retrieval in the biomedical domain", Informatics for health and social care. V.38, N.2, 2013, pp.150-170.

[2] T. Murray, S. Blessing, and S. Ainsworth, "Authoring tools for advanced technology learning environments: towards cost-effective adaptive, interactive, and intelligent educational software";

http://helios.hampshire.edu/ tjmCCS/atoolsbook/ chaptersV2/ChapterList.html.

[3] A. Gladun, J. Rogushina, J. Schreurs, and AbdelBadeeh Salem, "Ontology-based knowledge recognition in service-oriented virtual research environments Case: application in e-learning", Proc. of The 7th International Conference on Information Technology ICIT-2015, Al Zaytoonah University of Jordan, Amman, Jordan, 2015, pp.148-155.

[4] S. Staab, H. Schnurr, R. Studer, and Y. Sure "Knowledge Processes and Ontologies", IEEE Intelligent Systems, 2001, 16(1), pp. 26-34.

[5] T.R. Gruber, "The role of common ontology in achieving sharable, reusable knowledge bases"; http://www.cin.ufpe.br/ mtcfa/files/10.1.1.35.1743.pdf.

[6] A. Gómez-Pérez, A. Moreno, J. Pazos, and A. SierraAlonso, "Knowledge Maps: An essential technique for conceptualisation", Data \& Knowledge Engineering, V.33(2), 2000, pp.169-190.

[7] A. Kleshchev, and I. Artemjeva, "A Structure of Domain Ontologies and their Mathematical Models"; http://www.iacp.dvo.ru/es/

[8] V. Munteanu, C. Sandru, and D. Petcu, "Multi-cloud resource management: cloud service interfacing", Journal of Cloud Computing: Advances, Systems and Applications, 3(3), 2014

[9] S. Panica, M. Neagul, and C. Craciun, "Serving Legacy Distributed Applications by a Self-configuring Cloud Processing Platform", Proc. of the 6th IEEE International Conference on Intelligent Data Acquisition and Advanced Computing Systems: Technology and Applications (IDAACS"2011), 2011, pp.139-145.

[10] M.W. Wallin, and G. Von Krogh, "Organizing for open innovation: focus on the integration of knowledge", Organizational Dynamics, Vol.39 (2), 2010, pp.145-154.

[11] A. Zuiderwijk, K. Jeffery, and M. Janssen, "The necessity of metadata for linked open data and its contribution to policy analyses", Proc. of the International Conference for E-Democracy and Open Government CeDEM12, 2012, pp. 281-294.

[12] T. Berners-Lee, and J. Hendler, "Publishing on the Semantic Web”, Nature 410, 2001, pp.1023-1024.

[13] Web Ontology Working Group OWL Web Ontology Language Guide (2004); http://www.w3.org/TR/owlguide/.

[14] S. Ontanon, and E.Plaza, "Learning and joint deliberation through argumentation in multi-agent systems", Proc.of the 6th International Joint Conference on Autonomous Agents and Multi-Agent Systems AAMAS”07, Honolulu, Hawaii, USA, 2007, pp.971-978.

[15] A. Gladun, and J. Rogushina, "Mereological aspects of ontological analysis for thesauri constructing", Building and Environment, Nova Scientific Publishing, New-York, USA, 2009 , pp.198-212.

[16] R. Gruszczynski, "A short introduction to mereology";http://www.winterschool.eu/files/27-Short_ introduction_to_mereology.pdf. 
[17] A. Gladun, J. Rogushina, F. Garcia, R. MartínezBéjar, and J.T. Fernández-Breis "An application of intelligent techniques and Semanic Web technologies in elearning environments", Expert Systems with Applications, V.36, 2009, pp.1922-1931.

[18] S. Ontanon, and E. Plaza, "Learning and joint deliberation through argumentation in multi-agent systems", Proc.of the 6th International Joint Conference on Autonomous Agents and Multi-Agent Systems AAMAS"07, Honolulu, Hawaii, USA, 2007, pp.971-978.

[19] A. Gladun, J. Rogushina, "Distant control of student skills by formal model of domain knowledge", International Journal of Innovation and Learning (IJIL), InderScience Publishers, Vol. 7, No. 4, 2010, pp.394-411. 